\title{
A Comparative Study of Urban Road Traffic Simulators
}

\author{
Mustapha Saidallah, Abdeslam El Fergougui and Abdelbaki Elbelrhiti Elalaoui \\ Computer Science Department, ReSI team, Moulay Ismail University, Faculty of Science, 50000 Meknes, Morocco
}

\begin{abstract}
Road traffic management has become a worldwide concern. Several traffic simulators have been developed in order to contribute to solving traffic congestion problems. Comparative studies of simulators in this field of activity are concerned with the comparison of the simulation results with the results of the real situation; others are interested in the ability of certain platforms to simulate public transport systems. Our study aims purports to cover carryout of existing simulators in the sense that, on the one hand, poised in eleven major reviews of simulation platforms (commercial and open-source) the most used, given that the existing comparative studies do not cover all simulators we compared. On the other hand, our comparative study takes into consideration new criteria such as use wireless sensors and the ability of simulators to support GIS (Geographic Information System).
\end{abstract}

\section{Introduction}

Road traffic becomes increasingly a priority concern, especially in urban areas. We need a simulation of this traffic to better understand the problems of congestion in urban areas.

In general, the simulation is defined as a dynamic representation of some part of the real world through time. It is a tool that is widely used to test or evaluate a plan of action before its implementation.

Several studies have brought about the development of traffic simulation software, and also the comparison between these simulators is the subject of several articles.

The eleven selected simulators in our study are the most used and most mature, and we have compared them according to new criteria such as the use of wireless sensors which provides new opportunities for ITS (Intelligent Transport Systems), as well as the integration of Geographic Information Systems (GIS) which are effective tools for decision support in the field of road traffic, providing accurate data on the real world, and facilitating the establishment of the road network infrastructure.

The paper is organized as follows: Section 2 presents the different simulation software. Section 3 presents the related work on the comparison of urban road traffic simulators. The proposed criteria for the comparative study, a comparative table and a discussion of our comparison are listed in Section 4. In Section 5, a conclusion of our work will be provided.

\section{Simulation software}

Currently, there are several traffic simulation software, such as SUMO, MATSim, MITSIMlab, AIMSUN, CORSIM, Paramics, SimTraffic, VISSIM, TRANSIMS, etc. Some programs focus on the behavior of the vehicle in detail, the others are not interested in because they insist much on the simulation of a wide area. In simulation area, several tools can simulate a large city like MATSim simulated traffic in Berlin and Zurich.

\subsection{AIMSUN (Advanced Interactive Microscopic Simulator for Urban and Non-Urban Networks)}

AIMSUN [1], available from TSS-Transport Simulation Systems (Spain), is able to reproduce the real conditions of traffic of any network transport. It is, among others, used to develop and test the traffic control systems, traffic management rules, access controls, the location of tolls, public transport networks, lanes, and can work together with the vehicle guidance systems, and other real-time applications. It has many advantages over other software on the market, it allows notably to model the different network models in the same simulation. AIMSUN is embedded in GETRAM, a simulation environment composed of a traffic network editor (TEDI), a network database, a module for performing simulation and an Application Programming Interface which aims at interfacing to other simulation or assignment models. Through an additional library of DLL functions, the GETRAM extensions, the model is capable of communicating with external user-defined applications such as real-time control logic. 


\subsection{ARCHISIM}

ARCHISIM [2], developed by the team Modeling and Simulation of the National Institute for Research on Transport and Safety (INRETS), is a behavioral simulation model, its implementation follows the multiagent concepts. Drivers of vehicles are simulated agents. Their functions are based on three main processes: perception, decision and action. In ARCHISIM, there are two types of simulated components: agents and objects. The agents will gather information, strategize and then send the information. The main simulation agents are the drivers and pedestrians. The objects are road signals for example (horizontal or vertical). Each simulated driver is an autonomous software agent which evolves in a virtual environment and interacts with the other agents of the simulation performing its goals according to its skills and the current situation.

\subsection{CORSIM (CORridor SIMulation)}

CORSIM [3], sponsored and developed by The Federal Highway Administration (FHWA), is a traffic simulation software for signal systems, road networks, and highway systems. It consists of an integrated set of two models that represent the environment of the entire traffic. NETSIM represents traffic on city roads. FRESIM represents traffic on roads and highways. CORSIM provides its own interface and driver software, separate from TShell. In addition to the user interface, the CORSIM driver provides access to a new output data processor. The output processor enables the user to accumulate user-selected statistics and summary data during multiple runs of CORSIM. It writes the collected data to an Excel workbook, a comma-separated file, and/or a tab separated file.

\subsection{MATSim (Multi-Agent Transport Simulation)}

MATSim [4] developed by the Polytechnic of Zurich, provides a set of tools to implement a very large simulation based agents. It is used to simulate traffic in Zurich (Switzerland), Berlin (Germany), Padang (Indonesia) and Toronto (Canada). It can simulate the traffic of a vast region throughout the day. But it is not interested in retail vehicle behavior. MATSim pursues an activity-based approach to demand generation. Unlike other transportation simulation packages MATSim is throughout agent-based and generates individual activity plans as input to the network loading rather than (timedependent) origin-destination matrices as typically used in dynamic traffic assignment.

\subsection{MITSIMLab (MIcroscopic Traffic SIMulation Laboratory)}

MITSIMLab [5], developed at MIT (Massachusetts Institute of Technology), is a traffic simulator that assesses the impacts of alternative designs traffic management systems, information systems for travelers, public transport operations, and various ITS (Intelligent
Transport Systems) strategies at the operational level and contributes to their further refinement. It can evaluate systems such as advanced systems for traffic management and road guidance systems. The role of MITSIMLab is to represent "the world". Traffic and network elements are represented in detail in order to capture the sensitivity of traffic flows to the control and routing strategies. MITSIMLab is an open-source application where its core models have been written in $\mathrm{C}++$ and are fully available. It has been successfully applied in several traffic and research studies in the USA, the UK, Sweden, Italy, Switzerland, Japan, Korea, Malaysia and Portugal.

\subsection{Paramics (Parallel Microscopic Simulation)}

Paramics [6], marketed by Quadstone Paramics (UK), is fully scalable and designed to handle such diverse scenarios such one intersection with a highway congested or modeling of an entire city traffic system. It helps give a realistic representation of the landscape, with $2 \mathrm{D}$ and $3 \mathrm{D}$ visualization. It takes into account the buses, trams and also simulates pedestrians. It is used in different traffic patterns (urban, inter-urban, tunnel, port, and parking) and in many countries. A Paramics model is represented by a combination of "nodes, links and other associated objects" to replicate real life geometry constraints. Upon release from an "origin zone", each vehicle attempts to complete its journey towards a "destination zone" whilst being bounded by physical and dynamic vehicle parameters. Through the use of micro simulation, Paramics allows users to simulate individual vehicle movements to predict future travel pattern behavior as a result from a change in traffic volume or geometric road layout.

\subsection{SimTraffic}

SimTraffic [7], marketed by Trafficware (United States), is easy to use and its graphic interface provides the user with an encoding road network time significantly shorter than other models. Even inexperienced users can get a single operational network installation in a very short time. SimTraffic is a link-node model that uses driver behavior and vehicle performance algorithms to simulate individual vehicle movements through a network. The capabilities of SimTraffic were expanded in subsequent versions to model additional features such as freeways, ramps, and roundabouts. A significant disadvantage of SimTraffic is the lack of API functions or supporting detailed output of vehicle-state variable information and automated statistical analysis capabilities of other codes.

\subsection{SUMO (Simulation of Urban Mobility)}

SUMO [8] was developed at the German Aerospace Centre. In this simulator vehicles can move freely, collision between vehicles and accidents are simulated. Each vehicle has its own road and vehicle routing is dynamic. The vehicle behavior is taken into consideration such as changing lanes. Roads in SUMO are shown as a plurality of lanes. And the width of each lane is fixed. 
Also, the vehicle width is fixed. And it does not take into account the different types of vehicles. SUMO allows modelling of intermodal traffic systems including road vehicles, public transport and pedestrians. SUMO can be enhanced with custom models and provides various APIs to remotely control the simulation.

\subsection{TRANSIMS (TRansportation ANalysis and SIMulation System)}

TRANSIMS [9], developed at Los Alamos National Laboratory (USA), is an integrated set of tools to conduct analysis of a regional transportation system based on a cellular automaton. It uses a new modeling paradigm of individual travelers and their multi-modal transport (This is the carriage of goods by at least two successive modes of transport. Part of the journey can thus be carried by road, the other by sea, river or rail). TRANSIMS differs from previous travel demand forecasting methods in its underlying concepts and structure. These differences include a consistent and continuous representation of time; a detailed representation of persons and households; timedependent routing; and a person-based micro simulator. These advances are producing significant changes in the travel forecasting process. To date the TRANSIMS models have been tested with data from Dallas, Texas, and Portland (Oregon).

\subsection{TransModeler}

TransModeler [10], marketed by Caliper Corporation (USA). It can simulate all types of road networks, highways and areas of the city center, and can analyze multimodal networks. We can model and visualize the behavior of complex systems of circulation in an environment of two-dimensional or three-dimensional to illustrate and assess the dynamics of traffic flows, traffic lights, and overall network performance. TransModeler model freeway and urban networks in the same network with driver behavior models that are sensitive to complex inter-vehicle interactions in merging areas and intersections, model high occupancy vehicle (HOV) lanes, bus lanes and toll facilities to better understand their effects on traffic system dynamics and model evacuation plans and scenarios for response to natural disasters, hazardous spills, and other emergencies.

\subsection{VISSIM}

PTV VISSIM [11] developed by PTV (Planung Transport Verkehr AG) in Karlsruhe, Germany. It is part of the Vision Traffic Suite which also includes PTV Visum (analysis and forecast traffic) and PTV Vistro (optimization of the signal and the impact on traffic). VISSIM is one of the most used simulation software to simulate, evaluate and validate new transport policies and control systems. VISSIM is a multimodal simulator that allows users to define a full range of vehicle types including passenger cars, buses, trucks, and heavy and light rail vehicles as well as pedestrians and cyclists. One very important feature of VISSIM that sets it apart from others simulation models is its component object model (COM) programming interface. The $\mathrm{COM}$ interface allows users to develop and implement their own applications on the VISSIM network using computer programming language such as $\mathrm{C}++$, Visual Basic, or Python. The COM interface provides user-developed applications with an access to the network topology, signal control, path flows, and vehicle behavior enabling VISSIM to model complex control logic and sophistical transportation systems and components [12].

\section{Related work}

Several articles have focused on the comparison of urban road traffic simulators.

In [13] the authors have made a comparison of seven simulators according to their ability to simulate public transport according to certain criteria such as visualization, software category (commercial or opensource), the public transport infrastructure, the characteristics of public transport vehicles as well as passengers and pedestrians. The authors concluded that the simulators, AIMSUN, VISSIM and Paramics, offer the ability to import aerial images that can be used as background and as a guide in the development of road networks. 3D views offered in VISSIM and AIMSUN are better than Paramics. In Paramics, vehicles appear as 3D blocks without distinct characteristics. However, in VISSIM and AIMSUN, vehicles appear with distinct characteristics. In some simulation software, special attention was paid to the driver settings. For example, VISSIM offers different car-following or lane-changing models. Although VISSIM, AIMSUN and Paramics allow the simulation of bus circuit, only VISSIM simulates disembarkation and embarkation of passengers from the left side of roads. Therefore, only VISSIM allows bus stops on the left side or the right side of the road. The occupancy rate of the bus and the vehicle's capacity can also be defined in VISSIM and Paramics, while the number of doors in buses is only recognized in VISSIM.

In [14] the authors studied the two simulators VISSIM and CORSIM based on an urban network in the North Bund area, Hongkou District, Shanghai, China. They concluded that the use of the software, publishing and network configuration in CORSIM are easier. Due to the existence of different simulation mechanisms, CORISM simulation results can be transmitted quickly, while VISSIM configuration provides an easy interface with a separate output file to the simulation results. While for the calibration effort, the software provides multiple calibration parameters to allow the simulated network to reproduce the real situation. VISSIM is more appropriate for large intersections with broadband traffic, while CORSIM is good for modeling unsaturated intersections. VISSIM's simulation results are closer to the real situation.

The article [15] presents a comparison of seven simulators based on criteria such as visualization, expansion of the simulator and it is oriented agent. The classification of simulators based on their ability to 
simulate cars, buses, trucks, trains, bicycles and pedestrians, as the management of the sensors (such as traffic lights, electromagnetic loops, cameras, etc). The article found that MITSIM and SUMO do not have 3D visualization. VISSIM, Paramics and AIMSUN have a realistic $3 \mathrm{D}$ visualization which is very useful for presenting real-world scenarios. MITSIM does not provide expansion capabilities, but since the simulator is open-source researchers can modify and extend its core. Each simulator has advanced features, for example, VISSIM has parameters allowing the flexibility of its functions, Paramics is suitable for use of resources on distributed machines, AIMSUN provides various options for its extension, SUMO has a flexible architecture, and MAS -T2er Lab. and ITSUMO are oriented agent.

The aim of the research conducted in the article [16] was to provide a comparison of three selected platforms (SUMO, VISSIM and TRANSIMS) on a fragment of a real urban network (in Poland). In general, it seems that the model SUMO had too low capacity compared to the capacity of the actual network, but on the other hand, it is difficult to assess the capabilities of VISSIM and TRANSIMS were consistent with, or better than the actual network. However, despite some differences in quantitative measurements, effects similar to the spread of the traffic flow (appearance of network bottlenecks and traffic jam effects) were observed in all three models.

The article [17] offers a comparative analysis of fourteen simulators focusing on the simulation of traffic at highways, congested urban networks and adaptation of simulators to ITS (Intelligent Transport Systems) applications. This article revealed that AIMSUN, CORSIM and VISSIM are suitable for clogged arteries and highways, but AIMSUN is less suited compared to others. The AIMSUN characteristics are favorable to the creation of large urban and regional networks, but it requires difficult coding. The models AIMSUN, Paramics, INTEGRATION and CORSIM seem most suitable to ITS applications.

\section{Comparison of simulators discussion}

Our comparative study is based on a set of criteria chosen so as to draw conclusions about some features of the compared simulators, as well as understanding of their functioning.

\subsection{Comparison criteria}

\subsubsection{Simulation Model}

In the field of simulation of road traffic, there are three different models depending on the detail of the simulation.

Microscopic models: These models simulate the characteristics and interactions between individual vehicles. They produce essentially the trajectories of vehicles moving across the network. The processing logic includes algorithms and rules that describe how vehicles move and interact. It also includes acceleration, deceleration, lane changes and overtaking maneuvers.
Mesoscopic models: These models simulate individual vehicles, to this end, traffic is represented by small groups of traffic entities, whose interactions are described in a medium level of detail.

Macroscopic models: These models simulate the flow of traffic. They consider traffic characteristics (speed, flow and density) and their relationships. These models are making on the conservation equations of flows and traffic disturbances that spread in the traffic system. Consequently, they can be used to predict the spatial and temporal congestion that is caused by the traffic demand or incidents in a road network.

\subsubsection{Software Category}

We can classify traffic simulation software into two categories: open-source or commercial. The open-source designation or "open source code" applies to software whose license specifically meets the criteria established by the Open Source Initiative; that is to say, the possibilities of free redistribution, access to source code and create derivative works. While a commercial software or proprietary means software that does not allow legally or technically, or by any other means whatsoever, to simultaneously perform four software freedoms which are running the software for any type of use, to study its source code (and therefore access to the source code), the distribution of copies, as well as modification and thus the source code improvement.

\subsubsection{System}

There are two types of systems: discrete (variable changing at regular intervals of time) and continuous (variables change continuously).

\subsubsection{Visualization}

Visualization can be two-dimensional (2D), threedimensional (3D) or both. 3D visualization allows to be closer to the real world. Also it gives details of the simulation (eg the vehicle height, better visibility of traffic lights).

\subsubsection{Infrastructure}

The road network is the basis of any simulation. It includes roads, intersections (intersection, roundabout, etc). In this criterion we will evaluate the difficulty or ease of coding of the road network to be studied (complex junctions, etc) according to three criteria: easy coding, coding with an average difficulty and difficult or hard coding. As well as the coding flexibility of various elements of the infrastructure that may encode (expressways, multivalued panels, etc) according to three criteria: flexible, limited or very limited.

\subsubsection{Vehicles and pedestrians}

The evaluation of the vehicles will be done on several levels, firstly the type (car, truck, motorcycle, etc), 
secondly the dimensions (length, width, height), thirdly the priority (priority cars: police car, firefighters, ambulances, etc). As for pedestrians, we will assess whether the simulator supports pedestrian or not. Each simulator will also be evaluated on the ability to simulate the public transport vehicles such as buses and trams.

\subsubsection{Scope area}

The scope area is the maximum area that the simulator can simulate. In this criterion we will compare simulators for their ability to simulate traffic in a city, a region or an entire country.

\subsubsection{Detectors}

The simulators use sensors such as electromagnetic loops and cameras, for example to reckon the number of vehicles in the queue or manage traffic speeds. In this criterion we will classify the simulators supported by the type of sensor, wired such as the electromagnetic loop formed of 3 or 4 turns of copper wire embedded in the floor, or wireless sensor such as MICA2 [18], using waves to communicate. Wireless sensors have several advantages such as simplicity of implementation, low cost of installation and maintaining.

\subsubsection{GIS (Geographic Information System)}

In this criterion we will assess whether the simulator offers the possibility to import maps from geographic information systems to encode road network, using the following three options: yes, partially or not.

\subsection{Comparison and discussion}

Based on the criteria mentioned above, we have classified the traffic simulator as shown in Table 1 below.

The comparative table shows that only AIMSUN and TransModeler simulate three models at the same time, while others are only microscopic simulators.

VISSIM, SUMO, MATSim and AIMSUN simulate traffic continuously, by opposition to ARCHISIM, CORSIM, Paramics and TRANSIMS which use a discrete system. For MITSIMLab, SimTraffic and TransModeler we could not specify the system used owing to the lack of this information in the literature and in the user guides.

VISSIM and SimTraffic offer an easy coding of the road network, unlike AIMSUN, ARCHISIM and SUMO require difficult or heavy coding. But concerning the coding flexibility in the various infrastructure elements, AIMSUN, Paramics and VISSIM are more flexible than the other simulators.

The most commercial simulators support the type and size of the vehicle, as well as taking into considerations the pedestrians and emergency vehicles such as ambulances and police cars. They have the opportunity to simulate the public transport vehicles such as buses and trams, in contrast to open-source simulators.

All simulators use wired sensors. Additionally AIMSUN, Paramics and VISSIM use wireless sensors which are more efficient and cheaper.

AIMSUN, MATSim, TransModeler and VISSIM support GIS, while other simulators support them partially or not at all. MATSim is the only open-source simulator that uses GIS.

Table 1. Comparative table of traffic simulators.

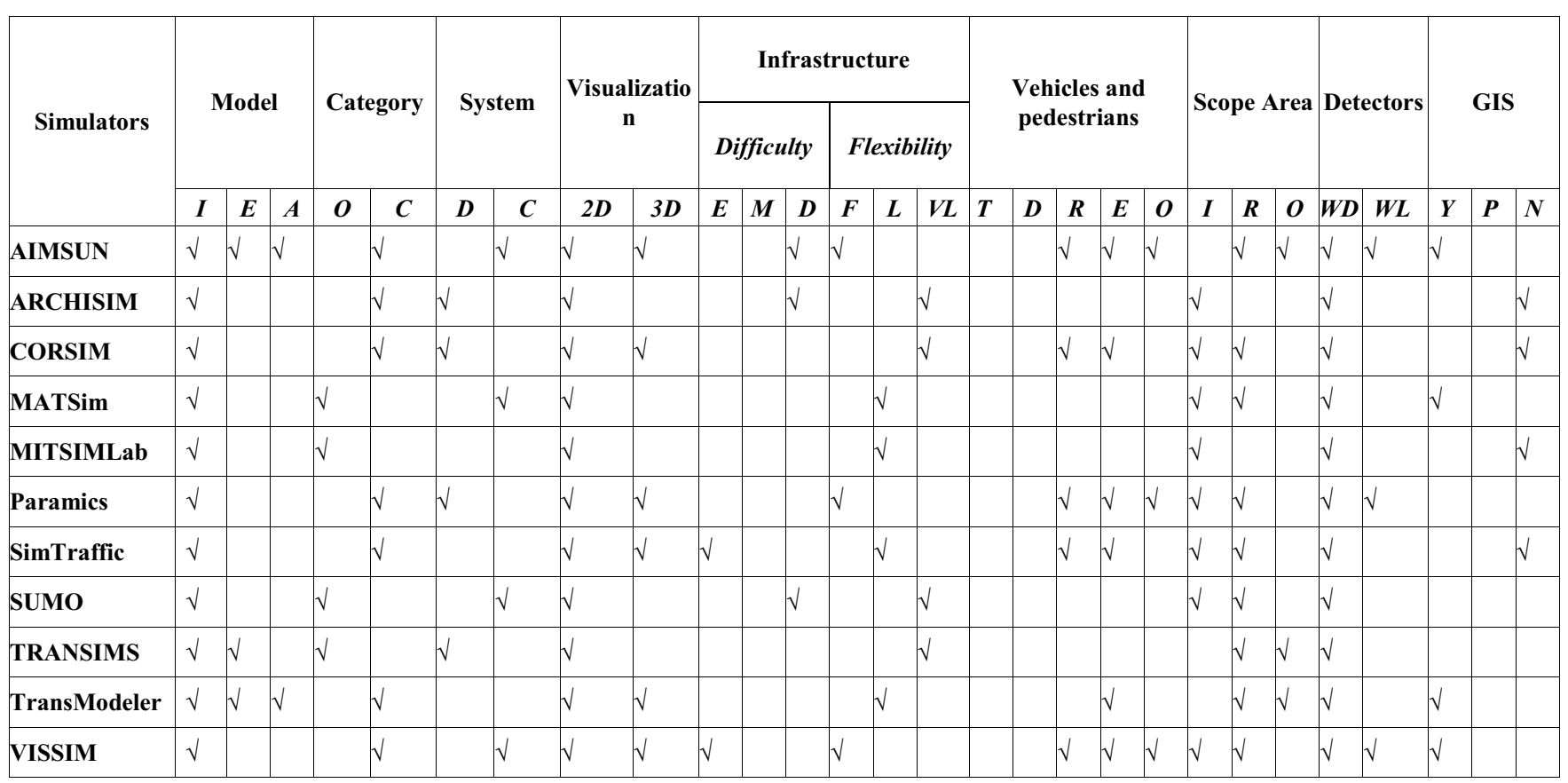


Table 2. Legend.

\begin{tabular}{|c|c|c|c|}
\hline \multicolumn{2}{|c|}{ Criteria } & Abbreviation & Signification \\
\hline \multicolumn{2}{|l|}{ Model } & $\begin{array}{l}\mathrm{I} \\
\mathrm{E} \\
\mathrm{A}\end{array}$ & $\begin{array}{l}\text { Microscopic } \\
\text { Mesoscopic } \\
\text { Macroscopic }\end{array}$ \\
\hline \multicolumn{2}{|l|}{ Category } & $\begin{array}{l}\mathrm{O} \\
\mathrm{C}\end{array}$ & $\begin{array}{l}\text { Open-source } \\
\text { Commercial }\end{array}$ \\
\hline \multicolumn{2}{|l|}{ System } & $\begin{array}{l}\mathrm{D} \\
\mathrm{C}\end{array}$ & $\begin{array}{l}\text { Discrete } \\
\text { Continuous }\end{array}$ \\
\hline \multicolumn{2}{|c|}{ Visualization } & $\begin{array}{l}2 \mathrm{D} \\
3 \mathrm{D}\end{array}$ & $\begin{array}{l}\text { Two-dimensional } \\
\text { Three-dimensional }\end{array}$ \\
\hline \multirow{2}{*}{$\begin{array}{l}\text { Infra- } \\
\text { structure }\end{array}$} & Difficulty & $\begin{array}{l}\mathrm{E} \\
\mathrm{M} \\
\mathrm{D}\end{array}$ & $\begin{array}{l}\text { Easy } \\
\text { Medium } \\
\text { Difficult }\end{array}$ \\
\hline & Flexibility & $\begin{array}{c}\mathrm{F} \\
\mathrm{L} \\
\mathrm{VL}\end{array}$ & $\begin{array}{l}\text { Flexible } \\
\text { Limited } \\
\text { Very Limited }\end{array}$ \\
\hline \multicolumn{2}{|c|}{ Vehicles and pedestrians } & $\begin{array}{l}\mathrm{T} \\
\mathrm{D} \\
\mathrm{R} \\
\mathrm{E} \\
\mathrm{O}\end{array}$ & $\begin{array}{l}\text { Type } \\
\text { Dimension } \\
\text { Priority } \\
\text { Pedestrian } \\
\text { Other vehicles (Bus } \\
\text { and Tram) }\end{array}$ \\
\hline \multicolumn{2}{|c|}{ Scope Area } & $\begin{array}{l}\mathrm{I} \\
\mathrm{R} \\
\mathrm{O}\end{array}$ & $\begin{array}{l}\text { City } \\
\text { Region } \\
\text { Country }\end{array}$ \\
\hline \multicolumn{2}{|l|}{ Detectors } & $\begin{array}{l}\text { WD } \\
\text { WL }\end{array}$ & $\begin{array}{l}\text { Wired sensor } \\
\text { Wireless sensor }\end{array}$ \\
\hline \multicolumn{2}{|l|}{ GIS } & $\begin{array}{l}\mathrm{Y} \\
\mathrm{P} \\
\mathrm{N}\end{array}$ & $\begin{array}{l}\text { Yes } \\
\text { Partially } \\
\text { No }\end{array}$ \\
\hline
\end{tabular}

\section{Conclusion}

The urban road traffic simulators are an vital tool to simulate and evaluate any planned change road network, in order to improve its functioning.

This paper provides an overview of eleven different traffic simulation platforms most used, and a comparison based on their different characteristics to provide a map that can be used as a decision support tool to select the simulator more suited to a need or as a reference for the study and development of new traffic simulators.

We can conclude that the open-source simulators do not simulate wireless sensors. It will be interesting to develop an open-source simulator integrating wireless sensors or integrate this feature in one of the existing open-source simulators.

The same thing for supporting GIS, type of vehicles and pedestrians, we can integrate these features to opensource simulators, or develop a new simulator integrating these features.

\section{References}

1. Casas, J., Ferrer, J. L., Garcia, D., Perarnau, J., \& Torday, A. (2010). "Traffic simulation with Aimsun". In Fundamentals of traffic simulation (pp. 173-232). Springer New York.

2. Bonte, L., Espié, S., \& Mathieu, P. (2006). "Modélisation et simulation des usagers deux-roues motorisés dans ARCHISIM." JFSMA, 6, 17.

3. Halati, A., Lieu, H., \& Walker, S. (1997). "CORSIM-corridor traffic simulation model." In Traffic congestion and traffic safety in the 21st century: Challenges, innovations, and opportunities.
4. "MATSim | Multi-Agent Transport Simulation", Matsim.org, 2016. [Online]. Available: http://www.matsim.org/. [Accessed: 29- May- 2016].

5. Yang, Q., \& Koutsopoulos, H. N. (1996). "A microscopic traffic simulator for evaluation of dynamic traffic management systems." Transportation Research Part C: Emerging Technologies, 4(3), (pp. 113-129).

6. Cameron, G. D., \& Duncan, G. I. (1996). "PARAMICS_Parallel microscopic simulation of road traffic." The Journal of Supercomputing, 10(1), (pp. 25-53).

7. Husch, D., \& Albeck, J. (2000). SIMTRAFFIC 5.0 User Guide for Windows.

8. Behrisch, M., Bieker, L., Erdmann, J., \& Krajzewicz, D. (2011, October). "SUMO-Simulation of Urban MObility." In The Third International Conference on Advances in System Simulation (SIMUL 2011), Barcelona, Spain.

9. Smith, L., Beckman, R., \& Baggerly, K. (1995). TRANSIMS: Transportation analysis and simulation system (No. LA-UR--95-1641). Los Alamos National Lab., NM (United States).

10. "TransModeler Traffic Simulation Software", Caliper.com, 2016. [Online]. Available: http://www.caliper.com/transmodeler/. [Accessed: 29- May- 2016].

11. Fellendorf, M., \& Vortisch, P. (2010). "Microscopic traffic flow simulator VISSIM." In Fundamentals of Traffic Simulation (pp. 63-93). Springer New York.

12. VISSIM 5.10 COM Interface Manual, PTV Germany, (2009).

13. Ghariani, N., Elkosantini, S., Darmoul, S., \& Ben Said, L. (2014, May). "A survey of simulation platforms for the assessment of public transport control systems." In International Conference on Advanced Logistics and Transport (ICALT), (pp. 8590). IEEE.

14. Sun, D. J., Zhang, L., \& Chen, F. (2013). "Comparative study on simulation performances of CORSIM and VISSIM for urban street network." Simulation Modelling Practice and Theory, 37, (pp. 18-29).

15. Kokkinogenis, Z., Passos, L. S., Rossetti, R., \& Gabriel, J. (2011). "Towards the next-generation traffic simulation tools: a first evaluation." In 6th Iberian Conference on Information Systems and Technologies (pp. 15-18).

16. Maciejewski, M. (2010). "A comparison of microscopic traffic flow simulation systems for an urban area." Transport Problems, 5(4), (pp. 27-38).

17. Ratrout, N. T., \& Rahman, S. M. (2009). "A comparative analysis of currently used microscopic and macroscopic traffic simulation software." The Arabian Journal for Science and Engineering, 34(1B), (pp. 121-133).

18. "Wireless Measurement System, MICA2", Crossbow Technology, Inc.41 Daggett Dr.San Jose, CA 95134. 